\title{
Textbook of the Neurogenic Bladder. 3rd ed.
}

By Jacques Corcos, David Ginsberg, Gilles Karsenty. Boca Raton, FL: CRC Press; 2015. 839 pp. £162.35. ISBN: 9781482215540

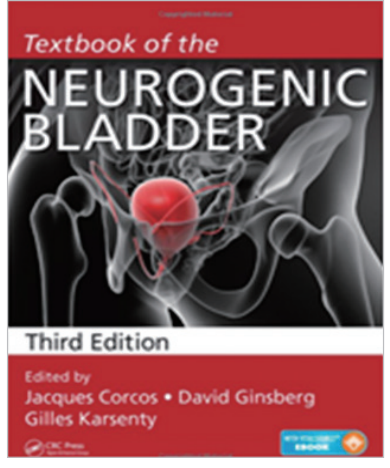

The symptoms and conditions resulting from a neurogenic bladder are one of the most commonly encountered clinical scenarios to those involved in managing lower urinary tract dysfunction. However, understanding the neuro-circuit and the various pathologies are considered difficult to learn and absorb. It is probably because the education and training involved are either too minimal or nearly nonexistence during residency. As the title states this is a textbook solely dedicated to the neurogenic bladder. It starts with the basic anatomy and functional pathology of the lower urinary tract and goes on to cover the methods involved in evaluating these patients. The evaluation section starts with pa- tient history and physical examination and reviews methods such as endoscopic, imaging, and electrophysiology exams. What also differentiate this book from others are the separate sections on meningomyelocele and sexual dysfunction. Many chapters with further detailed topic such as pregnancy in spinal cord injury will probably be difficult to find elsewhere. Although this book is mostly focused on adult conditions many discussions can still be applied to children and information specific to neurogenic bladder in children are also covered. This is a truly comprehensive one stop textbook for learning about the various types of neurogenic bladders.

Jang Hwan Kim Department of Urology, Yonsei University College of Medicine E-mail: jkim@yuhs.ac 